ESAIM: PROCEEDINGS, February 2007, Vol.16, 224-241

Eric Cancès \& Jean-Frédéric Gerbeau, Editors

DOI: $10.1051 /$ proc:2007004

\title{
NUMERICAL SIMULATION OF INSTATIONARY MAGNETOPLASMADYNAMIC THRUSTERS ${ }^{*, * *}$
}

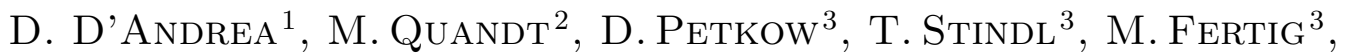 \\ M. Auweter-Kurtz ${ }^{3}$, C.-D. Munz ${ }^{2}$, S. Roller ${ }^{4}$, P. Adamidis ${ }^{4}$ And R. Schneider ${ }^{1}$
}

\begin{abstract}
In order to model rarefied plasma flows under conditions where continuum assumptions fail, a cooperation between IRS (Institute of Space Systems, University of Stuttgart), IAG (Institute for Aerodynamics and Gas Dynamics, University of Stuttgart), HLRS (High Performance Computing Center Stuttgart) and IHM (Institute for Pulsed Power and Microwave Technology, Research Center Karlsruhe) has been formed. Within the project "Modeling and Simulation on High Performance Computers", which is funded by the Landesstiftung Baden-Württemberg, a scheme for solving the Boltzmann equation for rarefied, non-continuum plasma flows is under development, making use of well known approaches from PIC (Particle in Cell) and DSMC (Direct Simulation Monte Carlo). The modeling will be explained in some detail and required computational resources will be estimated.
\end{abstract}

\footnotetext{
* We gratefully acknowledge the Landesstiftung Baden-Württemberg for funding the present work.

** T. Stindl wishes to thank the State of Baden-Württemberg and the Erich-Becker-Stiftung, Germany, for their financial support.

${ }^{1}$ Institute for Pulsed Power and Microwave Technology (IHM), Research Center Karlsruhe, D-76133 Karlsruhe, Germany

${ }^{2}$ Institute for Aerodynamics and Gas dynamics (IAG), University of Stuttgart, D-70550 Stuttgart, Germany

${ }^{3}$ Institute for Space Systems (IRS), University of Stuttgart, D-70550 Stuttgart, Germany

${ }^{4}$ High Performance Computing Center Stuttgart (HLRS), Nobelstr. 19, D-70569 Stuttgart, Germany
} 


\section{INTRODUCTION}

While PIC (Particle in Cell) methods allow the modeling of charged particle movement within electromagnetic fields, they do not usually take into account interactions between particles, i.e. collisions leading to energy and momentum exchange as well as to chemical reactions. Since these interactions can play an important role for the thrust and losses of electric propulsion systems, it is necessary to model them as well, using e.g. DSMC (Direct Simulation Monte Carlo) techniques. A cooperation between IRS (Institute of Space Systems, University of Stuttgart), IAG (Institute for Aerodynamics and Gas Dynamics, University of Stuttgart), HLRS (High Performance Computing Center Stuttgart) and IHM (Institute for Pulsed Power and Microwave Technology, Research Center Karlsruhe) has been formed to develop a hybrid PIC/DSMC scheme.

The main application for this scheme is the modeling of an instationary magnetoplasmadynamic (IMPD) thruster, also known as pulsed plasma thruster (PPT). Within the small satellite program of the IRS, a lunar satellite is under development. The satellite will be equipped with two electric propulsion systems. The main propulsion system will consist of a cluster of IMPD thrusters. The duration of a single pulse is of the order of $8 \mu \mathrm{s}$. The current of about $30 \mathrm{kA}$ allows an acceleration of the propellant mass bit leading to exhaust velocities of about $12 \mathrm{~km} / \mathrm{s}$, i.e. a specific impulse of approximately $1200 \mathrm{~s}$ [26]. Due to the instationary operation and the degree of rarefaction, no continuous partition function of the propelling plasma is to be expected.

A second application is the modeling of a combined electrodynamic tether/ electric propulsion (CETEP) system proposed by ESA [28]. The thrust generated by a traditional electrodynamic tether is limited by its current collection ability. TSS-1R (Tethered Satellite System), for example, was able to collect $0.3 \mathrm{~A}$ at about $800 \mathrm{~V}$ in flight using a passive metallic sphere. The PMG (Plasma Motor Generator) carried an active, i.e. plasma ejecting contactor and collected approximately the same current under $130 \mathrm{~V}$ bias [7]. For a coupled system, the tether links an ion thruster and its neutralizer. The electrons generated in the ion thruster are driven through the tether and released by the neutralizer. Thereby, the tether current is only limited by the number of electrons produced by the ion engine, resulting in a possible current of the order of 2-3 A (depending on the ion thruster used) and therefore a higher tether thrust, which is additionally amended by the thrust of the ion engine itself.

In order to model the physics, the PIC scheme developed by IHM [24,25] will be extended by models for intraspecies charged particle collisions and intermolecular reactions. Within the PIC code, the Vlasov-Maxwell equations are solved in order to describe the interaction between charged particles and electromagnetic fields. To model the exchange of momentum and energy as well as chemical reactions without consideration of Lorentz forces, a DSMC method based on the "LasVegas" code developed at IRS [19] is used. A newly developed FokkerPlanck solver, also using PIC techniques, is used to model collision relaxation of electrons in velocity space. The integration of these three models is expected to allow for an accurate prediction of the thrust of electric space propulsion systems operating far from continuum. Additionally, the necessity of a three dimensional and time accurate description, complex geometries and large numbers of particles requires efficient use of high performance computers. Therefore, optimization and parallelization of the code have to be addressed.

In Section 1, a description of the problem - finding a general solution to the Boltzmann equation - and the simplifications used are given. In Sections 2, 3, and 4, an overview of the methods used to model the various parts of the Boltzmann equation is given. In Section 5, conceptual aspects of managing the interplay of the three different models are discussed. First experiences concerning the performance of the code on high performance computers are considered in section 6 based on results obtained up to now. Finally, a short summary and an outlook are given in Section 7. 


\section{Boltzmann Equation}

From the microscopic point of view, a particle without internal degrees of freedom can be characterized by its mass and velocity. Combining all particles with equal mass $m_{i}$ within a volume element $d x_{1} d x_{2} d x_{3}$ defines a phase space distribution function

$$
f_{i}\left(\vec{x}, \vec{c}_{i}, t\right)
$$

where $\vec{c}_{i}$ is the particle velocity and $t$ is the time. The most general equation describing the change of the distribution function is the Boltzmann equation

$$
\frac{\partial f_{i}}{\partial t}+\vec{c}_{i} \cdot \nabla_{x} f_{i}+\frac{\vec{F}_{i}}{m_{i}} \cdot \nabla_{c} f_{i}=\left(\frac{\delta f_{i}}{\delta t}\right)_{\text {Coll }},
$$

which describes the change of the distribution function in time and phase space as a result of external forces and particle collisions. The term on the right-hand side of Eq. (2) represents the Boltzmann collision integral [10]

$$
\left(\frac{\delta f_{i}}{\delta t}\right)_{\text {Coll }}=\sum_{j} \int g\left(\vec{c}_{i}, \vec{c}_{j}\right) \sigma_{i j}(g)\left[f_{i}^{\prime}\left(\vec{c}_{i}^{\prime}\right) f_{j}^{\prime}\left(\vec{c}_{j}^{\prime}\right)-f_{i}\left(\vec{c}_{i}\right) f_{j}\left(\vec{c}_{j}\right)\right] d \Omega d \vec{c}_{j}
$$

which reflects the rate of change of $f_{i}$ due to collisions with respect to time. Here, the index $j$ runs over all "scattering" populations, $\vec{g}=\vec{c}_{i}-\vec{c}_{j}$ is the relative velocity, $\sigma_{i j}(g)$ is the differential scattering cross section between the particles of the species $i$ and $j$ and the element of solid angle $d \Omega$ is given by $d \Omega=\sin \theta d \theta d \phi$. Moreover, the prime refers to the value of a quantity after a collision and unprimed denotes the values before the collision. From the mathematical point of view, the Boltzmann equation is a very complicated integro-differential equation which can be used to determine the velocity distribution function. Up to now, a general solution of the Boltzmann equation to describe macroscopic problems is not possible. Therefore, several simplifications are necessary to compute solutions for the Boltzmann equation. In the case of highly non-neutral plasmas, the right-hand side of the Boltzmann equation (2) can be neglected. Then, the collective behavior of such an electrically non-neutral ensemble of charged particles is described by the Vlasov and Maxwell equations [24]. The corresponding numerical approach is known as the Maxwell-Lorentz model, which is based on Particle-inCell techniques. A brief review of this model is given in section 2. However, in order to model the physics of a PPT, collisions between particles of the same species as well as between different species have to be taken into account. An appropriate, lowest order approximation of the collision integral (3) leads to the Fokker-Planck model [30]. This approach is suitable for modeling intraspecies charged particle collisions and will be introduced in Section 3. A further approximation of the collision integral that allows the inclusion of interspecies reactions is presented in Section 4. Therein, the underlying DSMC method is applied to model ionization and recombination processes.

\section{Maxwell's Equations}

According to the law of dynamics for charged particles, the external force $\vec{F}_{i}$ in equation (2) is determined by the Lorentz force and depends on the velocity $\vec{c}_{i}$, the electric field $\vec{E}$, and the magnetic induction $\vec{B}$ :

$$
\vec{F}_{i}=q\left[\vec{E}(\vec{x}, t)+\vec{c}_{i} \times \vec{B}(\vec{x}, t)\right] .
$$

With the Lorentz force for charged particles (4), the Boltzmann equation (2) can be written in the collisionless kinetic formulation for the distribution function $f_{i}$ of the charged particles, which is the so called Vlasov equation [9]

$$
\frac{\partial f_{i}}{\partial t}+\vec{c}_{i} \cdot \nabla_{x} f_{i}+\frac{q_{i}}{m_{i}}\left(\vec{E}(\vec{x}, t)+\vec{c}_{i} \times \vec{B}(\vec{x}, t)\right) \cdot \nabla_{c} f_{i}=0 .
$$


In the terminology of hyperbolic partial differential equations, the general solution of (5) is given by its characteristics, which we call the Lorentz equations

$$
\begin{aligned}
\frac{d \vec{x}_{i}}{d t} & =\vec{c}_{i}, \\
\frac{d \vec{p}_{m, i}}{d t} & =\vec{F}_{i} .
\end{aligned}
$$

The relativistic momentum is given by $\vec{p}_{m, i}=m_{i} \gamma \vec{c}_{i}$ with the Lorentz factor $\gamma^{2}=1+\left(\vec{p}_{m, i}\right)^{2} /\left(m_{i} \overrightarrow{c_{L}}\right)^{2}$ where $\vec{c}_{L}$ denotes the speed of light.

The difficulties in solving the Lorentz equations arise from the fact that the electric field $\vec{E}$ and the magnetic induction $\vec{B}$ are not given explicitly. They have to be calculated at each time step in a self-consistent manner [11] from the full set of Maxwells equations

$$
\begin{aligned}
\frac{\partial \vec{E}}{\partial t}-c^{2} \nabla \times \vec{B} & =-\frac{\vec{j}}{\epsilon_{0}}, & & \text { Ampère's law, } \\
\frac{\partial \vec{B}}{\partial t}+\nabla \times \vec{E} & =0, & & \text { Faraday's law of induction, } \\
\nabla \cdot \vec{E} & =\frac{\rho}{\epsilon_{0}}, & & \text { Gauss' law, } \\
\nabla \cdot \vec{B} & =0, & & \text { absence of magnetic monopoles, }
\end{aligned}
$$

where the electric permittivity $\epsilon_{0}$ and magnetic permeability $\mu_{0}$ are related to the speed of light $c_{L}$ according to $\epsilon_{0} \mu_{0} c_{L}{ }^{2}=1$. For given charge and current densities $\rho$ and $\vec{j}$, the Maxwell equations describe the temporal and spatial evolution of the electric field $\vec{E}$ and the magnetic induction $\vec{B}$. With an integration over the entire range of momentum $\vec{p}_{m, i}$, the self consistent parts of the charge and current density $\rho, \vec{j}$ are obtained from [13]

$$
\begin{aligned}
\rho & =\sum_{i} q_{i} \int f_{i}(\vec{x}, \vec{p}, t) d^{3} p \\
\vec{j} & =\sum_{i} q_{i} \int \vec{c}_{i} f_{i}(\vec{x}, \vec{p}, t) d^{3} p .
\end{aligned}
$$

Up to now the description is exact in the sense that no numerical approximations are made. For the numerical realization of the Maxwell-Lorentz system, the Particle-In-Cell method is applied [14]. In the discrete case, the charge and current density can be obtained from

$$
\begin{aligned}
\rho^{*} & =\sum_{i} q_{i} \delta\left[\vec{x}-\vec{x}_{i}\right] \\
\vec{j}^{*} & =\sum_{i} q_{i} \vec{c}_{i} \delta\left[\vec{x}-\vec{x}_{i}\right]
\end{aligned}
$$

where $\delta$ denotes the standard Dirac function. For each grid node, all particles in the surrounded cells are considered. In order to determine the contribution of the particles, shape-functions are used to calculate $\rho$ and $\vec{j}$ at the grid nodes. With these charge and current densities the new electromagnetic fields are computed at these nodes and then interpolated to the local particle positions. To get these field values, the Maxwell equations have to be solved at each time step.

A direct consequence of the charge conservation equation

$$
\frac{\partial \rho}{\partial t}+\nabla \cdot \vec{j}=0 .
$$


and of the fact that the divergence of the curl equals zero for any differentiable vector field is that the divergence constraints (11) and (10) are satisfied for all times, if the initial values satisfy these relations. In this case, it would be sufficient to solve the hyperbolic evolution equations (8) and (9) only.

Unfortunately, in the simulation numerical errors may occur: The divergence of a curl may be zero up to some error terms only and interpolation errors in the particle treatment may be arise. This leads to small errors being introduced at each time step. If only the hyperbolic evolution equations are numerically solved, then these errors may increase and strongly falsify the solution. For a self-consistent movement of charged particles, Gauss' law (10) and the statement about the absence of magnetic monopoles (11) have to be coupled with Ampère's and Faraday's law. In the Generalised Lagrange Multiplier approach [23], two additional variables $\Phi(\vec{x}, t)$ and $\Psi(\vec{x}, t)$ are introduced into the Maxwell equations to couple the evolution equations for the electromagnetic fields (8) and (9) with their elliptical constraints (10) and (11), respectively. The coupling terms may be chosen such that a purely hyperbolic system can be formed. If the errors are zero it coincides with the original Maxwell equations. The Purely Hyperbolic Maxwell (PHM) equations system reads as

$$
\begin{aligned}
\frac{\partial \vec{E}}{\partial t}-c^{2} \nabla \times \vec{B}+\chi c^{2} \nabla \Phi & =-\frac{\vec{j}}{\epsilon_{0}} \\
\frac{\partial \vec{B}}{\partial t}+\nabla \times \vec{E}+\gamma \nabla \Psi & =0 \\
\nabla \cdot \vec{E}+\frac{1}{\chi} \frac{\partial \Phi}{\partial t} & =\frac{\rho}{\epsilon_{0}} \\
\nabla \cdot \vec{B}+\frac{1}{\gamma c^{2}} \frac{\partial \Psi}{\partial t} & =0
\end{aligned}
$$

where the dimensionless positive parameters $\chi$ and $\gamma$ represent the transportation coefficients for the local errors $\Phi$ and $\Psi$. These new variables $\Phi(\vec{x}, t)$ and $\Psi(\vec{x}, t)$ define two additional degrees of freedom and couple the divergence conditions (10), (11) to the evolution equation (8), (9).

This correction technique ensures that the divergence errors arising from the div curl as well as from the charge conservation violation within an electromagnetic PIC computation cannot increase and falsify the numerical simulation results. A decisive advantage of the proposed FV approach is that the explicit numerical methods used for the Maxwell equations in the time domain can be combined with a hyperbolic divergence correction in a straightforward manner, yielding a very efficient and highly flexible Maxwell solver module for PIC applications on unstructured grids and for parallel computing.

\subsection{Finite-Volume Method for the Maxwell Equations}

For this project, the presented PHM model is solved at this time with a finite volume scheme. In order to construct a finite-volume scheme, the extended Maxwell equation system (17)-(20) is written into the conservation form

$$
\frac{\partial \vec{u}}{\partial t}+\sum_{i=1}^{D} \frac{\partial \vec{f}_{i}}{\partial x_{i}}=\vec{g}
$$

where $\mathrm{D}=3$ in the present three-dimensional consideration. The vector $\vec{u}=\vec{u}(\vec{x}, t)$ consist of the components of the electric field, the magnetic induction and the two additional variables $\Phi$ and $\Psi$, and reads as

$$
\vec{u}=\left(E_{1}, E_{2}, E_{3}, \Psi, B_{1}, B_{2}, B_{3}, \Phi\right)^{T}
$$

The source $\vec{g}$ term on the right-hand side of the time dependent conservation equation (21) contains the current and charge density and is written as

$$
\vec{g}=-\frac{1}{\epsilon_{0}}\left(j_{1}, j_{2}, j_{3}, 0,0,0,0,-\chi \rho\right)^{T} .
$$


The physical fluxes $\vec{f}_{j}(\vec{u})$ are determined from $\vec{f}_{i}(\vec{u})=\mathcal{K}_{i} \vec{u} ; i=1,2,3$. In view of our special purposes in charged particle simulation, a homogeneous and isotropic media inside the computational domain is assumed, where the permittivity and permeability are constant. With these assumptions the block-structured $8 \times 8$ matrices $\mathcal{K}_{i}$ have constant entries and are defined as

$$
\mathcal{K}_{i}=\left(\begin{array}{cc}
0 & c^{2} \mathcal{M}_{i} \\
\mathcal{M}_{i}^{T} & 0
\end{array}\right)
$$

with

$$
\mathcal{M}_{1}=\left(\begin{array}{cccc}
0 & 0 & 0 & \chi \\
0 & 0 & 1 & 0 \\
0 & 0 & -1 & 0 \\
\gamma & 0 & 0 & 0
\end{array}\right), \mathcal{M}_{2}=\left(\begin{array}{cccc}
0 & 0 & -1 & 0 \\
0 & 0 & 0 & \chi \\
-1 & 0 & 0 & 0 \\
0 & \gamma & 0 & 0
\end{array}\right), \mathcal{M}_{3}=\left(\begin{array}{cccc}
0 & 1 & 0 & 0 \\
-1 & 0 & 0 & 0 \\
0 & 0 & 0 & \chi \\
0 & 0 & \gamma & 0
\end{array}\right)
$$

\subsection{Transversal Electro-Magnetic (TEM) Test Problem}

To study the convergence behavior the analytically known test problem of the temporal evolution of wave propagation in a coaxial wave guide was chosen. For the computational domain we use a cuboid with the two opposite corners $(0.5 ;-0.5 ; 0.0)$ and $(1.5 ; 0.5 ; 1.0)$, which represents a rectangular section in a wave guide with a rotational axis in $\mathrm{z}$ direction, which also defines the direction of the wave propagation. In this test case, the longitudinal field components (i.e. $E_{z}, B_{z}$ ) to the z-axis are zero and constant in time, while the transversal values are time dependent. The analytical formulas of the TEM fields are given by

$$
\begin{aligned}
& \left(\begin{array}{c}
E_{x} \\
E_{y} \\
E_{z}
\end{array}\right)=E_{0}\left(\begin{array}{c}
\frac{x}{x^{2}+y^{2}} \\
\frac{y}{x^{2}+y^{2}} \\
0
\end{array}\right) \sin (k z-\omega t), \\
& \left(\begin{array}{c}
B_{x} \\
B_{y} \\
B_{z}
\end{array}\right)=\frac{E_{0}}{c}\left(\begin{array}{c}
\frac{-y}{x^{2}+y^{2}} \\
\frac{x}{x^{2}+y^{2}} \\
0
\end{array}\right) \sin (k z-\omega t),
\end{aligned}
$$

where the wave number $k$ is related to the frequency according to $\omega=k c$. For the numerical experiment we choose $\mu_{0}=\epsilon_{0}=c=1, E_{0}=1 \mathrm{Vm}^{-1}$ and $k=2 \pi / L$ and initialize the fields according to their analytical values at $t=0$. In order to avoid problems with specific boundary conditions we prescribe the exact solution in the ghost cells. 

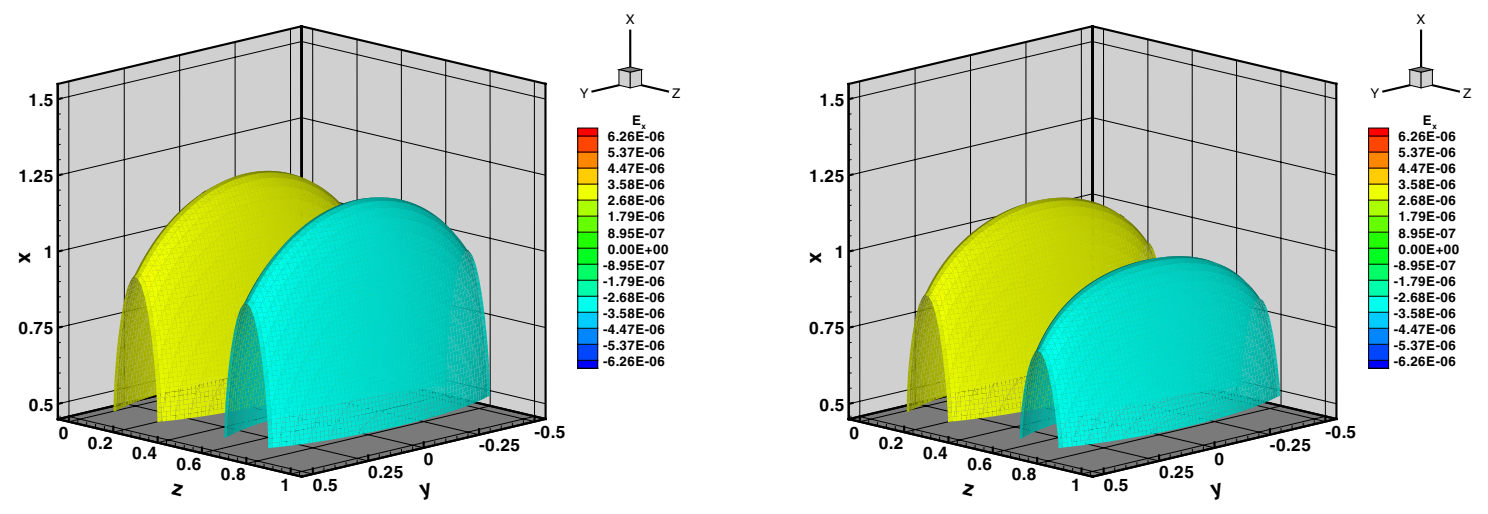

\begin{tabular}{|c|c|c|c|c|c|c|c|c|c|c|c|c|}
\hline \multicolumn{13}{|c|}{ Ex-Field L2ErrorNorm-TEM_Mode3D-10-Cube } \\
\hline & \multicolumn{2}{|c|}{$\begin{array}{l}\text { Space Discretization } \\
\text { Time Discretization } \\
\text { Boundary Condition }\end{array}$} & \multicolumn{2}{|c|}{$\begin{array}{l}\text { Maxwell-Solver } \\
\text { Runge-Kutta-Method } \\
\text { analytic }(\mu, \varepsilon, \mathbf{c}=1)\end{array}$} & \multicolumn{6}{|c|}{$\begin{array}{l}10 \\
10\end{array}$} & \multicolumn{2}{|c|}{ CFL $\quad 0,2$} \\
\hline Mesh & $\begin{array}{c}\text { Nbr. of } \\
\text { Cells }\end{array}$ & $\Delta \mathbf{x}$ & $\Delta t$ & max Error & L1-Error & L2-Error & $\begin{array}{c}\text { absolute } \\
\text { L2Error } \\
\text { Norm }\end{array}$ & $\begin{array}{l}\text { relative } \\
\text { L2Error } \\
\text { Norm }\end{array}$ & Oinf & 01 & 02 & $\mathrm{CPU}[\mathrm{s}]$ \\
\hline $\begin{array}{l}35 \times 35 \times 35 \\
40 \times 40 \times 40\end{array}$ & $\begin{array}{l}42875 \\
64000\end{array}$ & $1,43 \mathrm{E}-02$ & $\begin{array}{l}5,71 \mathrm{E}-03 \\
5,00 \mathrm{E}-03\end{array}$ & $3,30 \mathrm{E}-01$ & $\begin{array}{l}1,03 \mathrm{E}-01 \\
910 \mathrm{E}-02\end{array}$ & $\begin{array}{l}3,68 \mathrm{E}-07 \\
194 \mathrm{E}-07\end{array}$ & $1,26 \mathrm{E}-01$ & $1,73 \mathrm{E}-01$ & 0.9 & 09 & 09 & $\begin{array}{l}385 \\
712\end{array}$ \\
\hline $\begin{array}{l}45 \times 45 \times 45 \\
50 \times 50 \times 50\end{array}$ & $\begin{array}{c}91125 \\
125000\end{array}$ & $\begin{array}{l}1,11 \mathrm{E}-02 \\
1,00 \mathrm{E}-02\end{array}$ & $\begin{array}{l}4,44 \mathrm{E}-03 \\
4,00 \mathrm{E}-03\end{array}$ & $\begin{array}{l}2,65 \mathrm{E}-01 \\
2,41 \mathrm{E}-01\end{array}$ & $\begin{array}{l}8,15 \mathrm{E}-02 \\
7,38 \mathrm{E}-02\end{array}$ & $\begin{array}{l}1,10 \mathrm{E}-07 \\
6,57 \mathrm{E}-08\end{array}$ & $\begin{array}{l}9,99 \mathrm{E}-02 \\
9,06 \mathrm{E}-02\end{array}$ & $\begin{array}{l}1,38 \mathrm{E}-01 \\
1,25 \mathrm{E}-01\end{array}$ & $\begin{array}{l}0,9 \\
0,9\end{array}$ & $\begin{array}{l}0,9 \\
0,9\end{array}$ & $\begin{array}{l}0,9 \\
0,9\end{array}$ & $\begin{array}{l}1136 \\
1655\end{array}$ \\
\hline $55 \times 55 \times 55$ & 166375 & $9,09 \mathrm{E}-03$ & $3,64 \mathrm{E}-03$ & $2,21 \mathrm{E}-01$ & $6,74 \mathrm{E}-02$ & $4,13 \mathrm{E}-08$ & $8,29 \mathrm{E}-02$ & $1,14 \mathrm{E}-01$ & 0,9 & 0,9 & 0,9 & 2369 \\
\hline $65 \times 65 \times 65$ & 274625 & $7,69 \mathrm{E}-03$ & $3,08 \mathrm{E}-03$ & $1,90 \mathrm{E}-01$ & $5,75 \mathrm{E}-02$ & $1,83 \mathrm{E}-08$ & $7,08 \mathrm{E}-02$ & $9,77 \mathrm{E}-02$ & 0,9 & 1,0 & 0,9 & 4837 \\
\hline
\end{tabular}

FiguRE 1. $E_{x}$ field: initial cond. (left), calculated values after 5 periodes (right)

The numerical simulations are performed with 3 different kinds of elements and each with 6 shades of mesh resolution. In all calculations the computation has been carried out over 5 periods with a CFL restriction of 0.2. The iso-surfaces shown in Figure 1 are snapshots of the initial condition for the $E_{x}$ field at time $t=0$ and the numerically obtained one on the right side. The $x y$-plane at $z=0$ is the incoming plane of the waves which propagates in z-direction. The damping effect of this first order scheme is reflected in the lower value of the front wave. The calculated L2-Errors and the obtained order of convergence are shown below for a simulation with cube elements. Despite good results for a first order scheme this simulation shows clearly the need of a higher order scheme as the introduced numerical dissipation seems to be too high for practical use.

\section{A Diffusion Approach for Electron-Electron Collisions in PiC Simulations}

The electron energy distribution function (EEDF) in the plasma of a pulsed thruster is of large interest because this function controls - at least at the beginning - the degree of ionization of the plasma and to some extent the plasma chemistry. The shape of the EEDF is essentially determined through the competition between inelastic electron-neutral interactions and electron-electron - abbreviated by $(e, e)-$ collisions in the plasma. Highly energetic electrons are responsible for neutrals excitation or even for their ionization. These processes lead to a drastic reduction of the velocity of the scattered electrons (thermal or sub-thermal) and operate therefore as sinks of the electrons energy. The $(e, e)$-interactions are the driving force for the collisional relaxation, which pushes the electrons towards a Maxwellian velocity distribution and, furthermore, are responsible for the population of the high-energy tail of the Maxwellian. Finally, electrons from the high-energy regime of the Maxwellian are once again available for further excitation and ionization events, which closes the path of interplay. Clearly, since the EEDF determines many properties of the plasma, it is important to model $(e, e)$-collisions as realistic as possible. In the following, we describe the formulation that allows to include $(e, e)$-collisions into a PIC framework $[4,14]$ in a natural way. 


\subsection{Governing Equations}

A well-established and for our purposes suitable mathematical model for $(e, e)$-collisions is given by the Fokker-Planck (FP) equation

$$
\left(\frac{\delta f_{e}}{\delta t}\right)_{c o l}=-\sum_{p=1}^{3} \frac{\partial}{\partial c_{p}}\left[F_{p} f_{e}\right]+\frac{1}{2} \sum_{p, q=1}^{3} \frac{\partial^{2}}{\partial c_{p} \partial c_{q}}\left[D_{p q} f_{e}\right]
$$

This model describes the evolution of the electron distribution function $f_{e}=f_{e}(\vec{x}, \vec{c}, t)$ as a result of smallangle scattering of Coulomb point particles, and represents the lowest approximation order of the Boltzmann collision integral $[18,21,22,27,30]$. The key quantities to determine the coefficients of dynamical friction $F_{p}(\vec{x}, \vec{c}, t) \sim \partial \mathcal{H} / \partial c_{p}$ and diffusion $D_{p q}(\vec{x}, \vec{c}, t) \sim \partial^{2} \mathcal{G} / \partial c_{p} \partial c_{q}$ are the Rosenbluth potentials [21] given by

$$
\mathcal{H}(\vec{x}, \vec{c}, t)=2 \int_{-\infty}^{\infty} \frac{f_{e}(\vec{x}, \vec{w}, t)}{|\vec{g}|} d^{3} w
$$

and

$$
\mathcal{G}(\vec{x}, \vec{c}, t)=\int_{-\infty}^{\infty}|\vec{g}| f_{e}(\vec{x}, \vec{w}, t) d^{3} w
$$

where $\vec{g}=\vec{c}-\vec{w}$ is the difference between the velocity of the scattered-off electrons (test particles) and the velocity of the electrons that serve as scatterer (field particles). Clearly, the friction force $\vec{F}$ and the diffusion tensor $\mathbb{D}$ themselves depend on the velocity $\vec{c}$, and hence the FP model is in general a complicated non-linear problem that has to be solved numerically in an appropriate - namely, self-consistent - manner. Note that the appearance of the FP equation reveals that the $(e, e)$-collisions are modeled as a diffusion process that describes the short-time behavior of the system under consideration [12,15]. Moreover, this approach takes into account the long-range character of the Coulomb force in a natural manner, which is an advantage compared to "hard sphere" methods [3] whose strengths are the approximation of short-range interactions.

The FP equation (28) for the evolution of $f_{e}$ is equivalent to the stochastical differential equation (SDE) in the Itô sense $[12,16]$

$$
d \vec{C}(t)=\vec{F}(\vec{C}, t) d t+\mathbb{S}(\vec{C}, t) d \vec{W}(t)
$$

where $d \vec{W}(t)$ represents the three-dimensional Wiener increment and the matrix $\mathbb{S} \in \mathbb{R}^{3 \times 3}$ is related to the diffusion matrix according to $\mathbb{D}=\mathbb{S}^{T}$. As indicated, both quantities $\vec{F}$ and $\mathbb{S}$ now depend on the stochastical variable $\vec{C}=\vec{C}(t)$, which will be identified later as the velocity of a single (macro) electron. Hence, the use of the Langevin-type SDE (31) fits in a remarkable way into the standard PIC approach [24], which is one basic concept of the present code development.

\subsection{Numerical Methods and Results}

The key quantities for the numerical solution of the Langevin-type SDE (31) - which is solved in the context of the Fokker-Planck module [2,8] - are the velocity dependent friction and diffusion coefficients at each time step $t=t_{n}$. In order to compute these coefficients, we apply a PIC-type method in velocity space $(\vec{c}$-space). This method represents a self-consistent numerical description and fits in excellent manner into the complete concept of our hybrid PIC/DSMC code development. Schematically, a typical PIC-cycle is depicted in Fig. 2, which has to be executed for each grid cell of the spatial computational domain. 


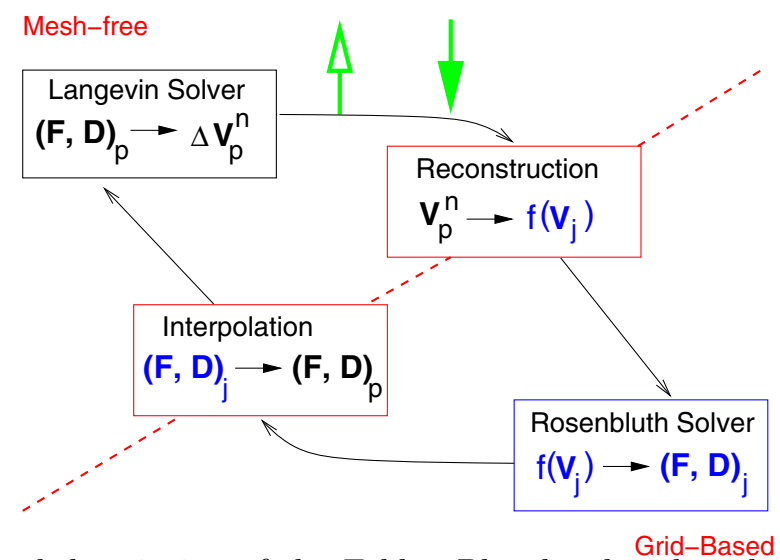

FIGURE 2. Schematical description of the Fokker-Planck solver based on the PIC method in velocity space.

A peculiarity of the PIC-method consists in the fact that a part of the calculations is performed on the grid-based computational domain (Rosenbluth-Solver) while for the other part the grid-free, continuous domain is appropriate (Langevin-Solver). Such a procedure assumes that at the interfaces "continuous/discrete" (Reconstruction) and "discrete/continuous" (Interpolation) suitable techniques are available to manage the data exchange.

In the context of the present paper we skip the discussion of the reconstruction and interpolation schemes, which are well established and reviewed, for instance in [8]. In the following, we first study the quality, property and reliability of the approximation characteristics of Rosenbluth and Langevin building blocks. Afterwards, we will focus on the assessment of the complete Fokker-Planck module which is used for the self-consistent computation of the friction and diffusion coefficients arising from the diffusive treatment of intra-species collision.

\subsubsection{Rosenbluth Solver.}

The central task of this building block is to provide the grid-based Rosenbluth potentials and their derivatives from which the friction and diffusion coefficients for the test particles are determined. In order to be free of any model assumption - like isotropic distribution of the field particles - we apply Fourier transformation techniques to compute the integrals in velocity space [8]. In essence, the friction and diffusion coefficient in compact form are given by

$$
F_{p}(\vec{c})=8 \pi i \kappa_{e e} \mathcal{F}^{-1}\left\{\frac{k_{p}}{k^{2}} \hat{f}_{e}(\vec{k})\right\}
$$

and

$$
D_{p q}(\vec{c})=8 \pi \kappa_{e e} \mathcal{F}^{-1}\left\{\frac{k_{p} k_{q}}{k^{4}} \hat{f}_{e}(\vec{k})\right\},
$$

respectively, where $\kappa_{e e}$ is a constant, $\hat{f}_{e}(\vec{k})$ represents the Fourier transform of the velocity distribution function $f_{e}(\vec{c})$ and $\mathcal{F}^{-1}\{\ldots\}$ denotes the inverse Fourier transformation of the arguments in the curly braces. To evaluate the occurring Fourier integrals we apply discrete Fourier transformation (DFT) methods, which represent special cases of the continuous transform [5]. For the practical examination of the DFT and its inverse we implement the highly efficient fast Fourier algorithm introduced by Cooley \& Tukey [5,6,29]. To demonstrate the quality and property of the coded Cooley \& Turkey algorithm, we simulate the approximation path consisting in the computation of the transform $\hat{f}_{e}(\vec{k})$, the subsequent multiplication of this result with $\vec{k}$-space functions and the final inversion of the expressions seen in the braces of (32) and (33) to get the desired coefficients. 

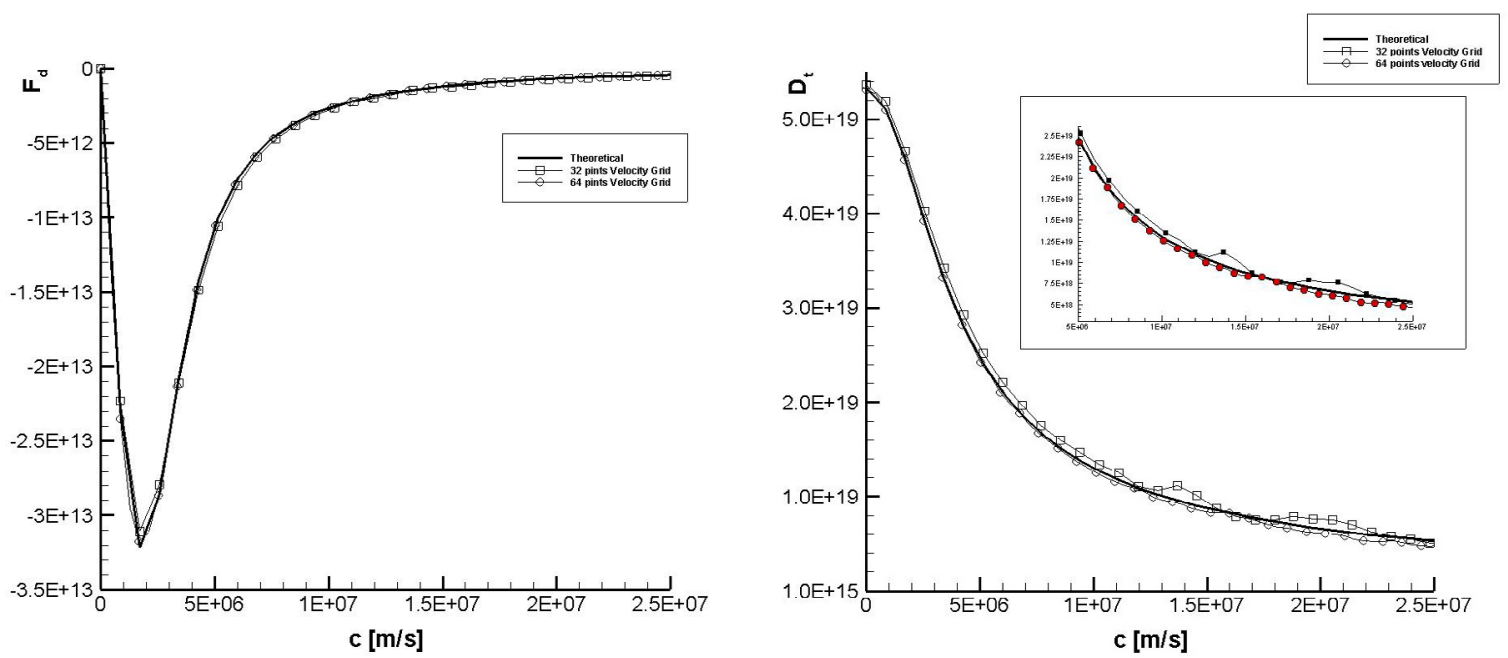

Figure 3. a) Fiction coefficient as a function of velocity obtained with the Fourier approach (squares: $N_{G}=32$ and circles: $N_{G}=64$ grid points) and the analytical solution (full line) b) Transversal diffusion coefficient as function of velocity computed with the discrete Fourier approach (squares: $N_{G}=32$ and circles: $N_{G}=64$ grid points) and analytical solution (full line)

The numerical experiment is initialized by a Gaussian velocity distribution $f_{e}(\vec{c})$ on the grid, where the thermal velocity $v_{\mathrm{th}}$ is adjusted for a temperature of $T_{e}^{\star}=10 \mathrm{eV}$. The results of the experiment (open symbols) and the analytical solution (full line) are seen in the Figures 3, where the friction and transversal diffusion coefficients are plotted. It is obvious from these Figures that the Rosenbluth solver produces very accurate approximations of the analytical results for both discretizations of the velocity grid $\left(N_{G}=32\right.$ : open squares; $N_{G}=64$ : open circles). However, we observe the expected tendency of the finer grid to better resolve especially the high-energy regime of the coefficients. The results obtained with the Rosenbluth solver recommend this building block for the application of self-consistent friction and diffusion coefficients computing arising from Fokker-Planck treatment of collisions.

\subsubsection{Langevin Solver.}

If the friction and diffusion coefficients are known for the current velocity $\vec{C}_{p}^{n}$ of the $p^{\text {th }}$ particle, then the new particle position in the $\vec{c}$-space can be evaluated solving the Langevin-type equation (31), which corresponds to the FP equation (28). This SDE must be solved in a numerical way whereas we use, for example, the Euler scheme

$$
\vec{C}_{p}^{n+1}=\vec{C}_{p}^{n}+\vec{F}\left(\vec{C}_{p}^{n}\right) \Delta t+\sum_{i=1}^{3}\left[\mathbb{S}\left(\vec{C}_{p}^{n}\right) \cdot \vec{e}_{i}\right] \Delta W_{n}^{i}
$$

which possesses a $\gamma=0.5$ strong order of convergence $[16,17]$. The approximated Wiener increment $\Delta W_{n}^{i}$ appearing in equation (34) is defined by $\Delta W_{n}^{i}=\sqrt{\Delta t} \eta_{n+1}^{i}$, where $\eta_{n+1}^{i} \sim \mathcal{N}(0,1)$ is a Gaussian random 
number with mean zero and variance one. Moreover, $\vec{e}_{i}$ is the $i^{\text {th }}$ Cartesian unit vector and $\mathbb{S} \in \mathbb{R}^{3 \times 3}$ is the matrix of the standard deviation.
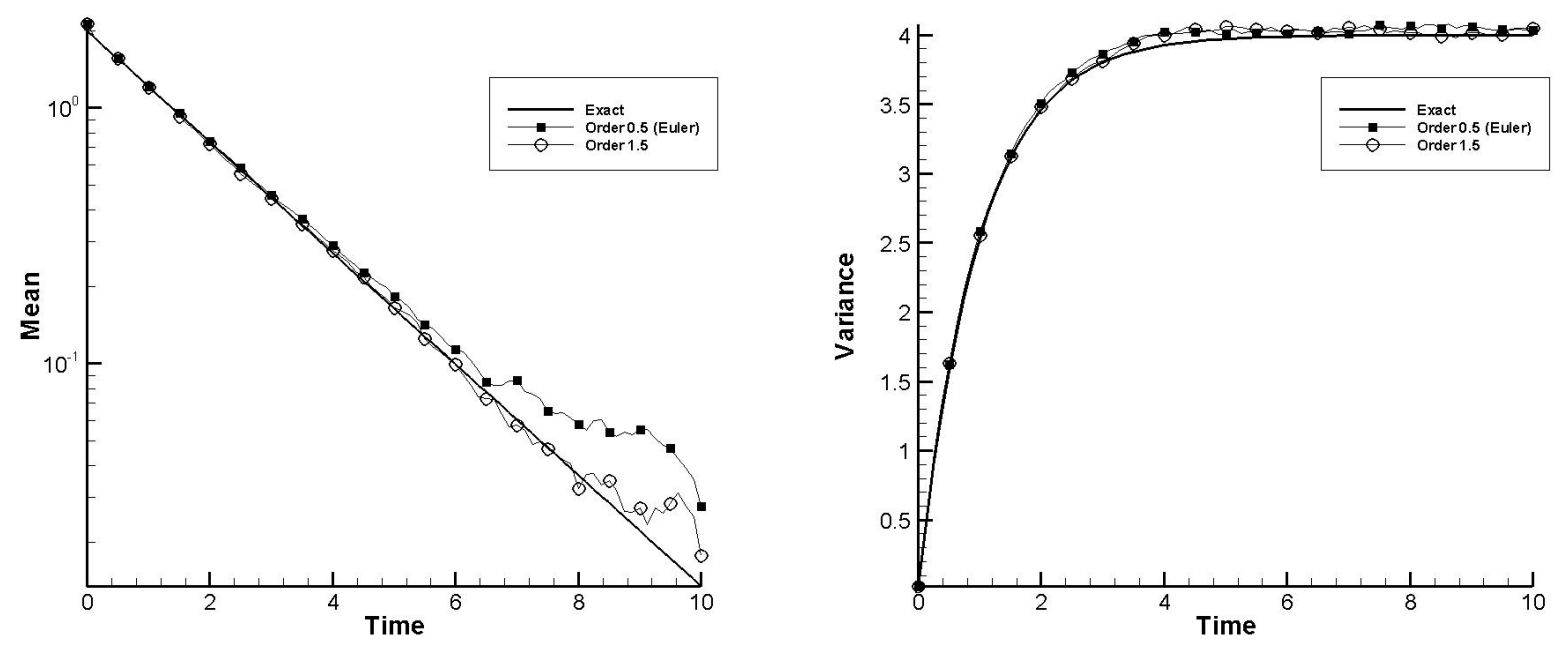

Figure 4. a) Temporal evolution of the mean value of the Ornstein-Uhlenbeck process. b) Comparison between the time-dependent variance Full line: exact solution, line with filled squares: Euler approximation $(\gamma=0.5)$, line with open circles: $\gamma=1.5$ strong order Itô-Taylor scheme

It should be noticed, that the order of the Euler scheme can easily be improved by adding an additional term of the Itô-Taylor expansion, which leads to an explicit $\gamma=1.0$ order scheme proposed by Milstein [16,17]. By means of the one-dimensional Ornstein-Uhlenbeck process $[12,16]$ the implementation of the solution procedure (34) was tested. This process is characterized through $F(C)=-a C(t)$ and $D(C)=\sigma^{2}$, where $a$ and $\sigma$ are constant values. Furthermore, the Ornstein-Uhlenbeck process was chosen because it possesses an analytical solution, wherefrom the mean and the variance can be calculated. Both quantities are represented in Fig. 4 as a function of the time. From these figures follows clearly that the Euler method (34) (line with squares) approximates the analytical results (full line) of mean and variance very satisfactorily. As expected, the expensive Itô-Taylor scheme with the strong convergence order of $\gamma=1.5$ (line with circles) shows an even better approximation behavior which follows especially out of the temporal development of the mean value (see Fig. 4). The good quality of the Euler approximation is confirmed once again through the result represented in Fig. 5a: there the numerically extracted (circle) with the analytical calculated (full line) velocity distribution function of the electrons is compared, that adjusted itself after $4 \cdot 10^{4}$ temporal iterations with $\Delta t=2.5 \cdot 10^{-4}$.

\subsubsection{Fokker-Planck Module.}

In conclusion the complete Fokker-Planck module, that, from a numerical point of view is based on the PICmethod in the velocity space (see Fig. 2), is tested. In addition we leave clear that the FP equation (28) can be written in spherical velocity coordinates [30], wherewith also the corresponding SDE is known $[1,8,12,16]$. Further, it can be shown, that the Maxwellian velocity distribution represents a solution of this equation [22]. Consequently we will start the numerical experiment with a Maxwellian distribution of the particle velocity

$$
h(c, 0)=g_{0}(c)=\frac{2}{\sqrt{2 \pi}} \frac{c^{2}}{v_{\mathrm{th}}^{3}} \exp \left(-\frac{c^{2}}{2 v_{\mathrm{th}}^{2}}\right)
$$



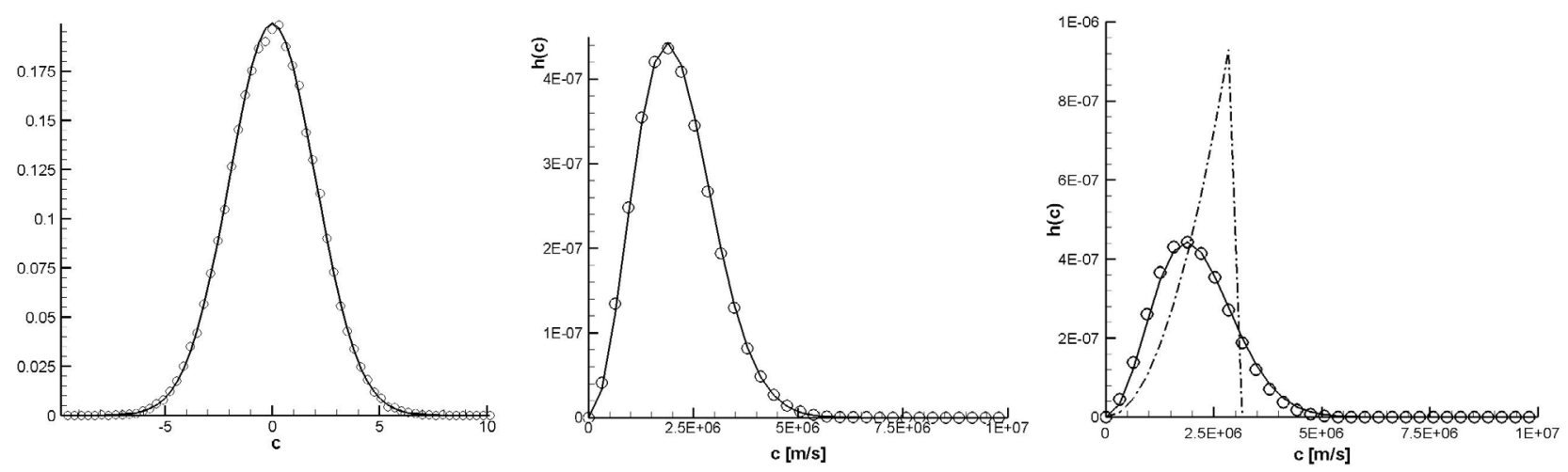

Figure 5. a) Comparison between the numerical (Euler scheme; open circles) and analytical (full line) particle distribution function recorded on the velocity grid after $4 \cdot 10^{4}$ temporal iterations b) Numerical (open circles) and exact (full line)velocity distribution after $2 \cdot 10^{4}$ temporal iterations. c) Temporal evolution of the initial velocity distribution (dashed-dotted line) to its equilibrium distribution function recorded after $10^{4}$ iterations (open circles). For comparison a Maxwellian is plotted (full line) corresponding to a temperature of $T_{e}^{\star}=10 \mathrm{eV}$

in the continuous mesh-free domain, and we expect that this distribution does not change in the course of the simulation with the Fokker-Planck-Solver. The result is depicted in Fig. 5b: there, the theoretical (Maxwellian) curve (full line) together with the numerical solution (open circles) is plotted after $2 \cdot 10^{4}$ iterations. Clearly, the temporal evolution of the distribution function obtained from the simulation stays very close to the Maxwellian shape. Moreover, this result demonstrates that also the high-energy tail is reproduced very well, although the resolution of this part is often critical due to the low number of particles.

A final numerical experiment is tailored to study the collisional relaxation of an arbitrary isotropic initial velocity distribution to its equilibrium from first principles. The shape of the initial velocity distribution (see Figure 5c dashed-dotted line) is given by

$$
h(c, 0)=g_{0}(c)=\left\{\begin{array}{ll}
3 \frac{c^{2}}{\tilde{v}^{3}}, & \text { for } c \leq \tilde{v} \\
0, & \text { for } c>\tilde{v}
\end{array},\right.
$$

where $\tilde{v}$ is set equal to $\tilde{v}=\sqrt{5} v_{\text {th }}$ with a thermal velocity $v_{\text {th }}$ corresponding to a temperature of $T_{e}^{\star}=10 \mathrm{eV}$. The temporal evolution of this initial distribution is monitored up to $2 \cdot 10^{4}$ cycles in time, where time step size is fixed equal to $\Delta t=0.177 \mathrm{ps}$ and the discretization of the velocity grid is performed by 64 grid points. The simulation result is seen in Figure 5c, where the initial profile (dashed-dotted line) and the equilibrium velocity distribution (open circles) - which is reached after $\sim 8400$ iterations [8] - recorded after $10^{4}$ temporal iterations are plotted. Additionally, the Maxwell velocity distribution corresponding to a temperature of $T_{e}^{\star}=10 \mathrm{eV}$ is seen in Figure 5 as a full line. Clearly, this plot demonstrates that the final state of the collisional relaxation process agrees very well with the analytical Maxwellian shape for $10 \mathrm{eV}$. It should be noticed, that the numerical simulation slightly underestimate the high-energy tail of the velocity distribution, which seemed to be an intrinsic property of the proposed collision model $[1,20]$.

\section{Modeling of Short Range Particle Interactions}

The losses within electromagnetically driven thrusters mainly depend on the density and distribution of neutral particles that could not have been ionized during the plasma discharge. For the characterization of those losses, particle collision models have to be taken into account. Unfortunately, no assumptions can be made with respect to the density and distribution of the neutrals due to very small particle densities and pulse 
durations - every single particle should be considered and tracked. Since the computational resources needed for such an approach are not available, two strategies are pursued: On one hand, real particles are merged to macro particles, which reduces the computational needs drastically, namely about several orders of magnitude. On the other hand, particle interactions are treated statistically instead of deterministically. This reduces the computational needs to a linear dependency on the particle number.

The short range particle interactions are treated using a DSMC (Direct simulation Monte Carlo) approach that was first discussed during the 1960s by Bird [3]. The main equation describing the behavior of rarefied and reacting gas flows is the full nonlinear gas kinetic Boltzmann equation (2). The collision term on the right hand side describes the collision based influences on the distribution function.

The DSMC approach is based on the determination of the total cross section $\sigma_{t, i j}$ of the particles $i$ and $j$ from the differential cross section, which ich part of the collision term on the right hand side of equation (2). The differential cross section depends on the relative particle velocity $g$ and the scattering angle $\chi$.

An intermolecular potential is being defined with the Hard Sphere (HS) model as the simplest approximation, see Figure 6. The potential is infinite for $r<d$ and 0 for $r>d$, the collision diameter is $d_{i j}=\left(d_{i}+d_{j}\right) / 2$, see
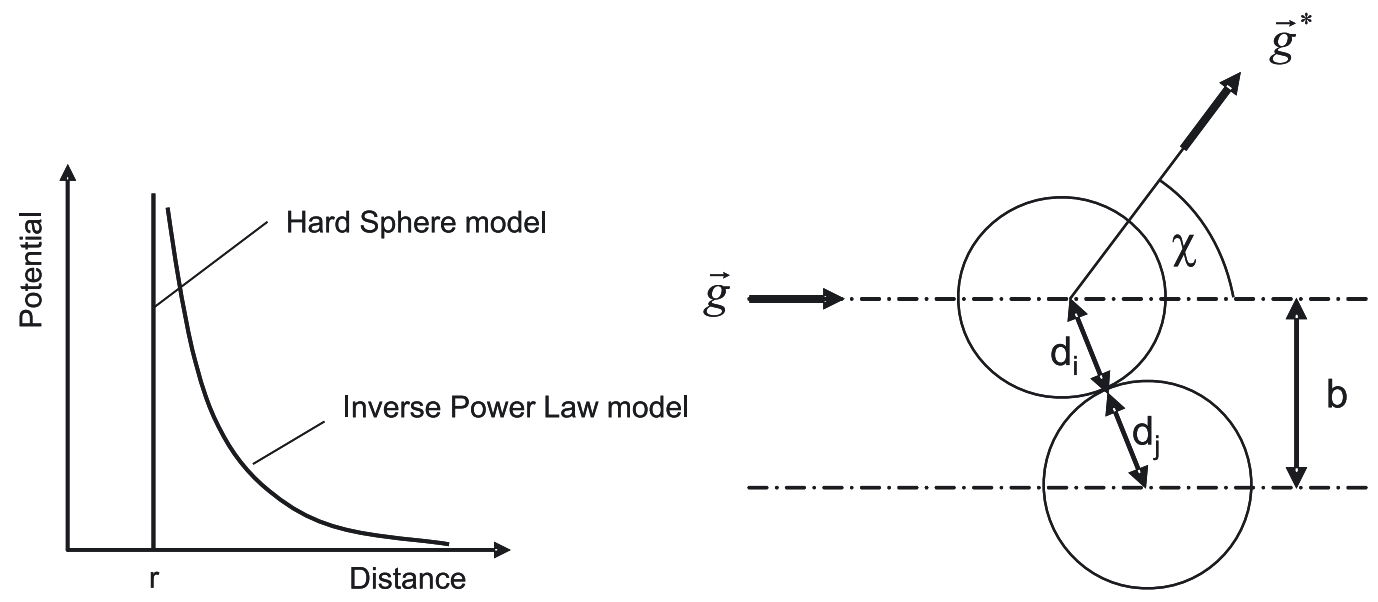

Figure 6. Mode of action for different potential models (left) and collision principle for HS model (right)

Figure 6. This simple model leads to isotropic scattering and the total cross section

$$
\sigma_{t, i j}=\pi d_{i j}^{2}
$$

which does not depend on $g$.

However, the isotropic scattering and the missing influence of the relative velocity on $\sigma$ put the advantage of the easy computable collision mechanics of the HS model into perspective. The accuracy can be increased by applying a more realistic exponential interaction potential, which is depicted in Figure 6 . The problem with this interaction model is that the cross section may become infinite and thus an appropriate determination of the collision frequency and the mean free path may be difficult. One way to address this problem is the use of Bird's VHS (Variable Hard Sphere) model which is based on the HS model. The scattering is isotropic again but the molecular diameter as a function of the temperature depends on the relative velocity now. The effective cross section of real molecules decreases as $g$ increases. The rate of decrease is directly related to the change of viscosity with temperature, namely $\mu \sim T^{\omega+1 / 2}$. Here, $\mu$ is the viscosity and $\omega$ is the index of the viscosity-temperature power law. For $\omega=0$, the HS model is obtained, for $\omega=1 / 2$, Maxwellian particles are simulated. The VHS model is relatively simple and yields more accurate results than the HS model. Thus, it became the most common cross section model for DSMC simulations and is used in the particle approach 
presented here. The resulting collision cross section is

$$
\sigma_{t}(g)=\pi d_{s, r e f}^{2}\left(\frac{2(2-\omega) k T_{s, r e f}}{\mu_{i j} g^{2}}\right)^{\omega} .
$$

The quantity $d_{s, r e f}$ is the reference diameter of the species $s$ at a reference temperature $T_{s, r e f}, \mu_{i j}=m_{i} m_{j} /\left(m_{i}+\right.$ $\left.m_{j}\right)$ is the reduced mass.

The collision probability

$$
P_{c}(g)=\beta g^{\phi}
$$

can be determined with the coefficients $\beta$ and $\phi$ as functions of the cell particle number, the number of real particles within a macro particle, the cell volume, the time step size and some species constants [19]. The collision probability (39) is compared with a random number $R \in[0,1]$. A collision occurs if $P_{c}>R$. Depending on the particle combination, further probabilities are calculated in respect to possible chemical reactions (dissociation, exchange and recombination) and relaxation of inner degrees of freedom. Due to this principle, the DSMC approach is stochastic in character.

The implemented neutral particle models are described in [19] in more detail and are tested by a reservoir simulation of an $\mathrm{O}-\mathrm{O}_{2}$ gas mixture. Only the main capabilities of the code are depicted here:

- the No-Time-Counter method is the central collision scheme,

- the Larsen-Borgnakke model is applied in order to distribute the collision energy to the inner degrees of freedom,

- rotation and vibration are modeled by a simple harmonic oscillator and its truncated version and

- particle collisions and reactions are treated by the Total-Collision-Energy model which can be extended to the Vibrationally-Favoured-Dissociation model in cases of known data.

After approximately 50.000 iteration steps, thermal equilibrium is reached. Chemical equilibrium is reached after approximately 300.000 iteration steps. The results are shown in Figure 7.
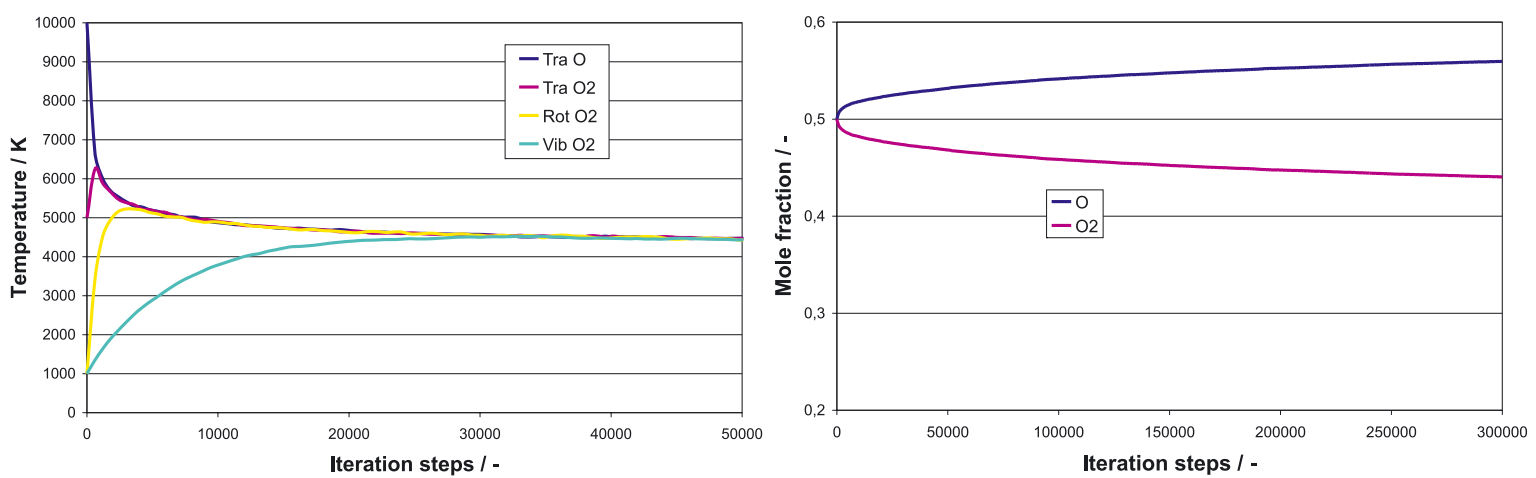

FiguRE 7. Thermal (left) and chemical (right) equilibrium after 50.000 and 300.000 iteration steps achieved

The code is developed in a way that every mon- and diatomic gas can be simulated since the proper species and reaction data is known. This simplifies the change to $C, F, C_{2}, F_{2}$ and $C F$.

The ionization energy is higher than the dissociation energy, thus it is assumed that only monatomic ions exist $\left(C^{+}+F^{+}\right)$. This shows that only an accurately determined plasma composition with a partially dissociated amount of neutrals leads to an accurate determination of the occuring losses mentioned above.

In the scope of DSMC, electrons are treated as particles as well. The implemented collision models based on the VHS model for the time being are to be improved and validated further. For the reaction models an exponential approach was chosen. The collision based boundary conditions will be implemented after the collision 
and reaction model of the charged particles has been validated.

Electron-Wall collisions: Electrons hitting the anode will be absorbed. In the case of a cathode the electrons will be reflected specularly. Further development may lead to a model extension regarding a diffuse reflection (Maxwell model) with partial energy accommodation (Cercignani-Lampis model).

Ion-Wall collisions: Ions colliding with the wall are reflected specularly. An extension regarding diffuse reflection with partial energy accomodation should be taken into account here as well. The collision of an ion with the cathode is modeled as a recombination followed by a re-emission of a neutral particle.

Neutral-Wall collisions: The collision of a neutral with a wall is modeled as a specular reflection independent on which kind of wall or electrode it is. Further development should also lead to the extended reflection model.

\section{Coupling Concept}

An important part of the project is the integration of the four aforementioned building blocks - that are a) the Maxwell solver, b) the Lorentz solver, c) the Fokker-Planck part, and d) the DSMC part - into one code. Part a) is a Finite Volume scheme, based on an (unstructured) computational grid. Parts b - d are particle based systems involving the grid only to determine possibly interacting particles. Therefore, inter- and extrapolation techniques form one main constituent of the coupling. The other are localization procedures to determine the position of a particle within the computational grid. Figure 8 shows the coupled iteration of one timestep.

Given a particle distribution within the computational domain, the charge and current densities define the electromagnetic eigenfields. Therefore, their values at the particle positions have to be assigned to node or cell values for the Maxwell solver, where they act as source terms. After completing the Maxwell step, the $\vec{E}$ - and $\vec{B}$-fields are known as cell averages in the Finite Volume context. They have to be evaluated at the particle positions to determine the Lorentz forces acting on the charged particles. Hence, the mean values have to be extrapolated from grid positions to particle positions with the desired order of accuracy. The Lorentz solver then determines the acceleration of the particles. Performing these steps forms the Particle-In-Cell framework.

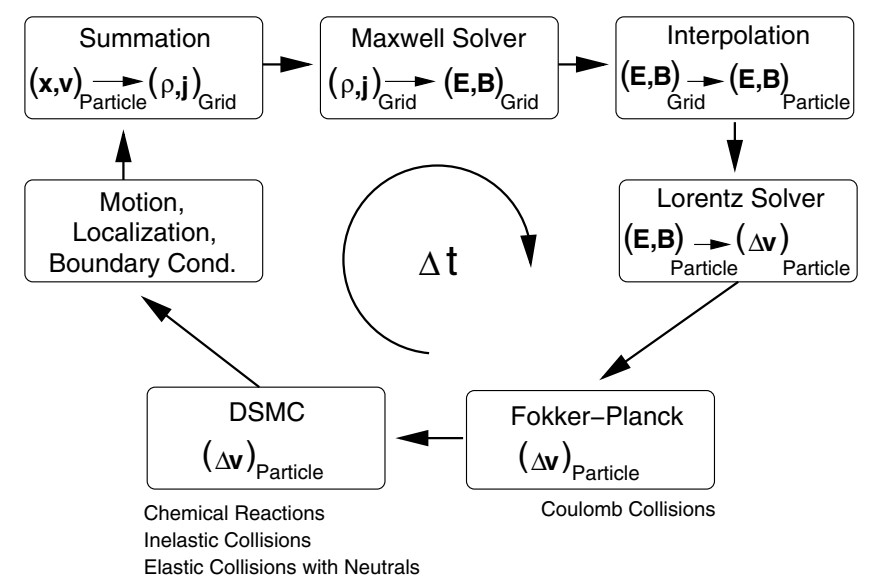

Figure 8. Coupling Circle for the integrated code

This scheme describes the movement of charged particles within an electric field and the recoupling of their eigenfields to the effective E- and B-fields. It does not account for interactions, neither collisions (intraspecies) nor chemical reactions (interspecies). Therefore the scheme is extended by the Fokker-Planck and DSMC blocks as described in sections $3-4$. They determine additional velocity changes of the charged as well as the uncharged particles. These steps, as well as the Lorentz solver, act on the same particle distribution, i.e. the distribution at the beginning of the time step, meaning that the different steps do not know about changes introduced by the 
other ones. Therefore these steps can be performed in parallel. Each step changes the velocity of the particles without actually moving them.

The movement of the particles is done in a separate pushing step. The particles are advanced using the simple Newtonian mechanics. Within this step, the new positions of the particles have to be localized on the grid to assign them to the grid cell they are in and/or the nearest barycenters or Gaussian integration points. This information is necessary for the application of correct boundary conditions as well as for the next step of the Maxwell solver.

Localization of particles is done in two different ways: If the previous position of the particle is known, the cross-products of the particle velocity vector with particle-to-node vectors are calculated to determine the side face through which the particle has left. Then, the corresponding neighboring cell is checked in the same way. This is an efficient procedure, suitable for vectorization and parallelization.

Newly emitted particles have to be localized differently, since no knowledge from a previous time step is available. In this case, the unstructured computational grid is overlayed by a cartesian-equidistant one. There, the search is done by dividing the position of the particle by the grid size $\Delta x$, yielding the correct structured cell for this particle. Then, only the unstructured cells contained in this structured cell have to be checked, until the correct one is found.

In order to reduce the computational time for particle localizations, especially of the newly emitted particles, all geometrical information about the unstructured-structured cell link is calculated in an initialization step prior to the time iteration procedure. Here, mapping matrices of structured grid cells and the contained unstructured cells have to be generated. These matrices are sorted in order to reduce the bandwidth and to obtain long loops for better optimization.

The particle push, including the localization of the particles within the grid as well as the application of boundary conditions, is the closing step of each time step, leading to a new particle distribution used in the following time step.

The numerical and implementational requirements of the four building blocks differ strongly, therefore the parallelization strategy is not self-evident. It has been decided to parallelize over the computational domain, i.e. to apply a domain decomposition method. Also, parallelization over the particles was considered. For the Lorentz solver, this would be possible, since all particles move independently. The movement is determined only by electric and magnetic forces acting on them. However, the Fokker-Planck and the DSMC step need information about neighboring particles to determine the interaction probabilities. For this, pairs of nearby particles have to be determined by random processes. Therefore, the particles cannot be considered independent of each other and it has to be guaranteed that these particles remain on the same processor. Thus, a grid based domain decomposition containing not only the cell values but also the information about all particles located in these cells is chosen as parallelization strategy. 


\section{Simulations on High Performance Computers}

The sections above clearly show the need for high performance computing. Three-dimensional calculations have to be performed on grids with approximately $10^{6}$ grid cells and the same amount of particles.

The Maxwell solver as an explicit Finite-Volume scheme is expected to vectorize well. This step has to be calculated before the particle based steps, which can then be calculated in parallel. These steps account for different physical phenomena: the Lorentz solver calculates the acceleration of charged particles, i.e. electrons and ions, due to electro-magnetic forces. In this step, the acceleration of each particle is calculated from the electro-magnetic forces, i.e. independent from the other particles. They can therefore be distributed over as many processors as available with MPI parallelization. The Fokker-Planck and the DSMC step on the other hand account for interactions of particles with each other. Here, neighbourhood relationships have to be known to define, which particles are close enough to interact. Domain decomposition is to be used where all particles belonging to the same domain remain on the same computational node. This guarantees that particles that are close to each other remain on the same node. Within each node, the loops over particles can then be parallelized by OpenMP. These steps require a shared memory machine.

Up to now, the integration of the different building blocks into one code is still in progress. Therefore, test calculations have been performed with the standard PIC scheme (i.e. Maxwell + Lorentz solver).

The PIC tests have been performed with approximately $10^{3}$ grid cells and 3.000 .000 particles. This calculation required $1418 \mathrm{sec} \mathrm{CPU}$ and $1616 \mathrm{MB}$ of memory to reach a simulation time of about $20 \mathrm{~ns}$. Increasing the number of grid cells by a factor of 10 in each spatial dimension, i.e. increasing the total number of grid cells from $10^{3}$ to $10^{6}$ requires not only a factor of 1000 more effort per timestep, but also $10 \times$ more timesteps to reach the same final computation time, due to the explicit time step restriction (CFL-condition). Therefore, a pure PIC calculation with $10^{6}$ grid cells containing $3 \times 10^{6}$ particles will require about 4000 CPU-hours for $20 \mathrm{~ns}$.

We expect about 5-10 complete thruster simulations and a lot of small calculations like the $10^{3}$ grid cells case above. In total we apply for about 100000 CPU hours. We expect to use 4-16 nodes per simulation.

\section{Summary AND OUTLOOK}

This paper describes the development of a numerical scheme that accounts for all effects that particles can undergo in a rarefied plasma. The integration of the different building blocks into one code is still in progress. Nevertheless, implementation is in advanced stadium and has to be validated in the nearest future. This concerns all parts of the code, from single building block to coupling and interaction up to performance optimization.

Another point to be addressed to is the second order in the interpolation and assignment steps: By making use of the gradients of the electro-magnetic fields, the second order interpolation is straight forward. This is implemented and currently undergoing validation tests. The advantage of this method is easy implementation, short CPU times at runtime and easy parallelization since no neighbouring information is needed. The disadvantage is the more complex implementation of the assignment of particle's charge to the cell centers when more than one particle is inside a cell.

As far as short range particle interactions, i.e. chemistry is concerned, it was shown in chapter 4 how and that the collision and reaction models for neutral mon- and diatomic species lead to thermochemical equilibrium. Currently, the chemical models are extended by ionization and the reversal recombination processes, which are necessary for a complete simulation of the performance of an IMPD thruster.

In the context of High Performance Computing (HPC), the load balancing in the parallel application will be the most challenging topic for the future, due to the different needs of the four building blocks.

This paper has shown a lot of special topics to be adressed in order to enable the integrated simulation of a complete IMPD thruster. 


\section{REFERENCES}

[1] B.J. Albright, D. Winske, D.S. Lemons, W. Daughton, and M.E. Jones. Quite simulation of Coulomb collision. IEEE Trans. Plasma Sci., 31:19-24, 2003.

[2] M. Auweter-Kurtz, M. Fertig, D. Petkow, T. Stindl, M. Quandt, C.-D. Munz, P. Adamis, M. Resch, S. Roller, D. D’Andrea, and R. Schneider. Development of a hybrid PIC/DSMC code. IEPC-2005-71, Proceedings of the 29th International Electric Propulsion Conference, Princeton, USA, 2005.

[3] G.A. Bird. Molecular Gas Dynamics and the Direct Simulation of Gas Flows. Clarendon Press, Oxford, 1994.

[4] C.K. Birdsall and A.B. Langdon. Plasma Physics via Computer Simulation. Adam Hilger, Bristol, Philadelphia, New York, 1991.

[5] E.O. Brigham. The Fast Fourier Transform and its Application. Prentice-Hall, Englewood Cliffs, 1988.

[6] J. W. Cooley and J. W. Tukey. An algorithm for the machine calculation of complex Fourier series. Math. Comput., 19:297-301, 1965.

[7] M.L. Cosmo and E.C. Lorenzini. Tethers in space handbook. The Smithsonian Astrophysical Observatory, Cambridge, Third Edition, 1997

[8] D. D'Andrea, C.-D. Munz, and R. Schneider. Modeling of electron-electron collisions for particle-in-cell simulations. FZKA $\mathbf{7 2 1 8}$ Research Report, Forschungszentrum Karlsruhe - in der Helmholtz-Gemeinschaft, 2006.

[9] R.C. Davidson. Theory of Nonneutral Plasmas. Benjamin, Reading, Massachusetts, 1974.

[10] D. Diver. A Plasma Formulary for Physics, Technology, and Astrophysics. Wiley-VCH Verlag, Berlin, 2001.

[11] M. Fedoruk, C.-D. Munz, P. Omnes, and R. Schneider. A Maxwell-Lorentz solver for self-consistent particle-field simulations on unstructured grids. Forschungszentrum Karlsruhe - Technik und Umwelt, FZKA 6115, 1998.

[12] C. W. Gardiner. Handbook of Stochastic Methods. Springer Verlag, Berlin, Heidelberg, New York, 1985.

[13] E. Halter, M. Krauß, C.-D. Munz, R. Schneider, E. Stein, U. Voß, and T. Westermann. A concept for the numerical solution of the maxwell-vlasov system. Technical Report FZKA 5654, FZKA, Karlsruhe, Germany, 1995.

[14] R. Hockney and J. Eastwood. Computer Simulation using Particles. McGraw-Hill, New York, 1981.

[15] J. Honerkamp. Stochastische Dynamische Systeme. VCH Verlagsgesellschaft, Weinheim, 1990.

[16] P.E. Kloeden and E. Platen. Numerical Solution of Stochastic Differential Equations. Springer-Verlag, Berlin, Heidelberg, New York, 1992.

[17] P.E. Kloeden, E. Platen, and H. Schurz. Numerical Solution of SDE Through Computer Experiments. Springer-Verlag, Berlin, Heidelberg, New York, 2003.

[18] N. A. Krall and A. W. Trivelpiece. Principles of Plasma Physics. McGraw-Hill, New York, 1973.

[19] M. Laux. Direkte Simulation verdünnter, reagierender Strömungen. PhD thesis, Institut für Raumfahrtsysteme, Universität Stuttgart, Germany, 1996.

[20] W. Manheim, M. Lampe, and G. Joyce. Langevin representation of Coulomb collision in PIC simulation. J. Comput. Phys., 138:563-584, 1997.

[21] M. Mitchner and C. Kruger. Partially Ionized Gases. Wiley, New York, 1973.

[22] D. Montgomery and D. Tidman. Plasma Kinetic Theory. McGraw-Hill, New York, 1964.

[23] C.-D. Munz, P. Omnes, and R. Schneider. A three-dimensional finite-volume solver for the Maxwell equations with divergence cleaning on unstructered meshes. Computer Physics Communications, 130:83 - 117, 2000.

[24] C.-D. Munz, R. Schneider, E. Sonnendrücker, E. Stein, U. Voß, and T. Westermann. A finite-volume particle-in-cell method for the numerical treatment of the Maxwell-Lorentz equations on boundary-fitted meshes. Int. J. Numer. Meth. Engng., 44:461-487, 1999

[25] C.-D. Munz, R. Schneider, and U. Voß. A finite-volume particle-in-cell method for the numerical simulation of devices in pulsed power technology. Surv. Math. Ind., 8:243-257, 1999.

[26] A. Nawaz, M. Auweter-Kurtz, G. Herdrich, and H. Kurtz. Investigation and optimization of an instationary MPD thruster at IRS. International Electric Propulsion Conference, Princeton, USA, 2005.

[27] D.R. Nicholson. Introduction to Plasma Theory. Wiley, New York, 1983.

[28] D. Nicolini and W.J. Ockels. Coupled electrodynamic tether/ electrostatic propulsion system. International Electric Propulsion Conference, Toulouse, France, 2003.

[29] W. H. Press, B. P. Flannery, S. A. Teukolsky, and W. T. Vetterling. Numerical Recipes. Cambridge University Press, Cambridge, 1987.

[30] M. Rosenbluth, W. MacDonald, and D. Judd. Fokker-Planck equation for an inverse-square force. Phys. Rev., 107:1-6, 1957. 